\title{
A DUALITY APPROACH TO DISCRETE TIME CONTROL THEORY
}

\author{
C. H. SCOTT and T. R. JEFFERSON
}

(Received 10 October 1977)

(Revised 23 January 1978)

\begin{abstract}
In this paper we use the theory of generalized geometric programming to develop a dual for a discrete time convex optimal control problem. This has interesting interpretational implications. Further it is shown that the variables in the dual problem are intimately related to the costate vector in the usual Maximum Principle approach.
\end{abstract}

\section{Introduction}

It is becoming increasingly apparent that discrete time systems are a useful way of modelling management decision making problems that are of a dynamic nature $[2,3]$. A common approach to discrete time optimal control theory, developed in conjunction with the aerospace and chemical process industries, is via the Discrete Maximum Principle which effectively decouples an $N$-stage problem to $N$ one-stage problems at the expense of introducing a complicated two-point boundary value problem. Alternatively, one may view a discrete time optimization problem as a mathematical program, albeit often of high dimension. In this paper, we adopt the latter viewpoint for the purpose of constructing a geometric dual to the original problem. Duals are often useful from a computational point of view as they may be easier to solve and used in conjunction with the primal (original) problem provide a powerful algorithmic stopping criteria. Further, one often obtains additional insight into the problem under consideration.

For the mathematical machinery necessary to generate the dual problem, we use the "unconstrained" version of generalized geometric programming [4]. In

(C) Copyright Australian Mathematical Society 1978

Copyright. Apart from any fair dealing for scholarly purposes as permitted under the Copyright Act, no part of this JOURNAL may be reproduced by any process without written permission from the Treasurer of the Australian Mathematical Society. 
Section 2, the required theory is summarized. In Section 3, we consider a linearly constrained convex discrete time optimal control problem and find its geometric dual. This admits an interesting reverse time interpretation.

Further, whereas the primal has mixed state-control variable-type constraints, the dual has only non-negativity constraints as inequality constraints. Mixed constraints pose considerable computational difficulties in the Maximum Principle approach [1], whereas non-negativity conditions may be routinely handled by penalty functions for example. We then particularize to a linear and a quadratic objective since these are both analytically tractable and of most interest in management science applications. Section 4 makes the connection between this theory and the Discrete Maximum Principle approach.

The theory may be further generalized to handle convex inequality constraints by using standard theorems of generalized geometric programming [4].

\section{Duality theory}

We consider an optimization problem of the form:

$$
\text { minimize } f(x), x \in C,
$$

subject to the constraints

$$
x \in \chi,
$$

where $\chi$ is a cone in $R^{n}$ and $f$ is a closed convex function with domain $C$ in $R^{n}$. This is termed the primal problem. We associate with the primal problem, another problem, called the dual problem, which is of the form:

$$
\text { minimize } g(y), y \in D,
$$

subject to the constraints

$$
y \in \chi^{*},
$$

where $\chi^{*}$ denotes the polar cone of $\chi$ in $R^{n}$ and $[g: D]$ is the conjugate transform of $[f: C]$ defined by

$$
g(y)=\sup _{x \in C}\left(x^{\mathbf{T}} y-f(x)\right)
$$

and

$$
D=\left\{y \in R^{n} \mid \sup _{x \in C}\left(x^{\mathbf{T}} y-f(x)\right)<+\infty\right\}
$$

T denotes a transpose and

$$
\chi^{*}=\left(y \mid x^{T} y \geqslant 0, \forall x \in \chi\right\} .
$$

We note that the primal and dual problems are essentially of the same form and hence are termed symmetric. At optimality, Peterson [4] has shown that the 
following relationships hold between the primal and dual optimal points, $x_{0}$ and $y_{0}$ respectively.

$$
\begin{gathered}
f\left(x_{0}\right)+g\left(y_{0}\right)=0, \\
y_{0} \in \partial f\left(x_{0}\right), \quad x_{0} \in \partial g\left(y_{0}\right), \\
x_{0}^{\mathrm{T}} y_{0}=0 .
\end{gathered}
$$

Here $\partial f\left(x_{0}\right)$ denotes the subgradient set of $f$ at $x_{0}$ and is defined by

$$
\partial f\left(x_{0}\right)=\left\{x^{*} \mid f(z) \geqslant f\left(x_{0}\right)+x^{* \mathrm{~T}}\left(z-x_{0}\right), \forall z \in C\right\} .
$$

In the case where $f(x)$ is a differentiable function, the subgradient is replaced by the gradient, that is, (9) may be written

$$
y_{0}=\nabla f\left(x_{0}\right), \quad x_{0}=\nabla g\left(y_{0}\right)
$$

where $\nabla$ denotes the gradient operator.

These optimality conditions allow an optimal point for one program to be calculated from an optimal point of the other.

\section{Duality in discrete time optimal control}

We consider an $N$-stage optimal control problem of the form:

$$
\operatorname{minimize} \sum_{i=0}^{N-1} c_{i}\left(x_{i}, u_{i}\right)
$$

subject to dynamics

given

$$
\begin{aligned}
x_{i+1}-x_{i} & =A_{i} x_{i}+B_{i} u_{i}, \quad i=0, \ldots, N-1, . \\
x_{0} & =a,
\end{aligned}
$$

and constraints

$$
x_{N} \in X_{N}=\left\{x_{N} \mid A_{N} x_{N}=0\right\}
$$

$$
E_{i} x_{i}+F_{i} u_{i} \leqslant b_{i}, \quad i=0, \ldots, N-1,
$$

where $x_{i} \in R^{n}$ are state variables, $u_{i} \in R^{m}$ are control variables, $c_{i}$ is jointly convex in $x_{i}$ and $u_{i}, b_{i} \in R^{p}, A_{i}, B_{i}, E_{i}$ and $F_{i}$ are appropriately dimensioned matrices at each stage $i$.

To invoke the duality theory of generalized geometric programming given in Section 2, we need to put the constraining equations (14), (15), (16) and (17) into a cone. Hence we associate with $a$, a vector variable $\alpha$ which is in turn restricted to a one-point domain $\{a\}$. Similarly we associate with $b_{i}$, a vector variable $\delta_{i}$, for all $i$, which is restricted to a one-point domain $\left\{b_{i}\right\}$, for all $i$.

The above problem may now be put into the form suitable for generalized geometric programming. This is,

$$
\operatorname{minimize} \sum_{i=0}^{N-1} c_{i}\left(x_{i}, u_{i}\right)
$$


subject to cone constraints

$$
\begin{aligned}
x_{0}-\alpha & =0, \\
x_{i+1}-\left(A_{i}+I\right) x_{i}-B_{i} u_{i} & =0, \quad i=0, \ldots, N-1, \\
-E_{i} x_{i}-F_{i} u_{i}+\delta_{i} & \geqslant 0, \quad i=0, \ldots, N-1,
\end{aligned}
$$

and the implicit domain-type constraints

$$
C=\left\{\left(x_{i}, u_{i}, \alpha, \delta_{i}\right) \mid x_{i} \in R^{n}, u_{i} \in R^{m}, \alpha \in\{a\}, \delta_{i} \in\left\{b_{i}\right\}, \forall i, x_{N} \in X_{N}\right\}
$$

The dual problem is given by taking the conjugate transform of equation (18) subject to the implicit constraints, equation (22), and then taking the polar cone to the cone constraints given by equations (19), (20) and (21). Hence the dual objective is given by (see equations (3) and (5))

$$
\begin{aligned}
\sup _{\left(x_{i}, u_{i}, \alpha, \delta_{i}\right) \in C}\left(\sum_{i=0}^{N} x_{i}^{\mathrm{T}} y_{i}+\sum_{i=0}^{N-1}\left(u_{i}^{\mathrm{T}} v_{i}+\delta_{i}^{\mathrm{T}} \gamma_{i}\right)+\alpha^{\mathrm{T}} \beta-\sum_{i=0}^{N-1} c_{i}\left(x_{i}, u_{i}\right)\right) \\
=\sup _{\substack{x_{i} \in R^{n} \\
u_{i} \in R^{m}, \forall i \\
x_{N} \in X}}\left(\sum_{i=0}^{N} x_{i}^{\mathrm{T}} y_{i}+\sum_{i=0}^{N-1} u_{i}^{\mathrm{T}} v_{i}-\sum_{i=0}^{N-1} c_{i}\left(x_{i}, u_{i}\right)\right)+\sup _{\alpha \in\{a\}} \alpha^{\mathrm{T}} \beta+\sup _{\delta_{i} \in\left\{b_{i}\right\}, \forall i} \sum_{i=0}^{N-1} \delta_{i}^{\mathbf{T}} \gamma_{i} \\
=\sum_{i=0}^{N-1} c_{i}^{*}\left(y_{i}, v_{i}\right)+a^{\mathrm{T}} \beta+\sum_{i=0}^{N-1} b_{i}^{\mathrm{T}} \gamma_{i},
\end{aligned}
$$

where $c_{i}^{*}\left(y_{i}, v_{i}\right)$ is the conjugate transform of $c_{i}\left(x_{i}, u_{i}\right), y_{i} \in R^{n}, v_{i} \in R^{m}$, $i=0, \ldots, N-1, \beta \in R^{n}, \gamma_{i} \in R^{p}, i=0, \ldots, N-1$, and $y_{N}=A_{N}^{\mathrm{T}} w \in R^{n}$. The polar cone of equations (19), (20) and (21) is given by the set of

where

$$
\left(y_{0}, \ldots, y_{N}, v_{0}, \ldots, v_{N-1}, \beta, \gamma_{0}, \ldots, \gamma_{N-1}\right)
$$

$$
\sum_{i=0}^{N} x_{i}^{\mathrm{T}} y_{i}+\sum_{i=0}^{N-1}\left(u_{i}^{\mathrm{T}} v_{i}+\delta_{i}^{\mathrm{T}} \gamma_{i}\right)+\alpha^{\mathrm{T}} \beta \geqslant 0
$$

for all $x_{0}, \ldots, x_{N}, u_{0}, \ldots, u_{N-1}, \alpha, \delta_{1}, \ldots, \delta_{N-1}$ satisfying equations (19), (20) and (21). A routine, though tedius calculation gives the following equations.

$$
\begin{array}{rlrl}
y_{i} & =z_{i-1}-\left(A_{i}^{\mathrm{T}}+I\right) z_{i}-E_{i}^{\mathrm{T}} q_{i}, & i=0, \ldots, N-1, \\
y_{N}=z_{N-1}, & \\
v_{i}=-B_{i}^{\mathrm{T}} z_{i}-F_{i}^{\mathrm{T}} q_{i}, & i=0, \ldots, N-1, \\
\beta=-z_{-1}, & i=0, \ldots, N-1 . \\
\gamma_{i}=q_{i} \geqslant 0, &
\end{array}
$$


Substituting equations (28) and (29) into the dual objective, equation (23), we obtain the following dual optimal control problem:

$$
\text { minimize } \sum_{i=0}^{N-1} c_{i}^{*}\left(y_{i}, v_{i}\right)-a^{\mathrm{T}} z_{-1}+\sum_{i=0}^{N-1} b_{i}^{\mathrm{T}} q_{i},
$$

subject to the constraints

$$
\begin{array}{rlrl}
y_{i} & =z_{i-1}-\left(A_{i}^{\mathrm{T}}+I\right) z_{i}-E_{i}^{\mathrm{T}} q_{i}, & i=0, \ldots, N-1, \\
y_{N} & =z_{N-1}=A_{N}^{\mathrm{T}} w, & & \\
v_{i} & =-B_{i}^{\mathrm{T}} z_{i}-F_{i}^{\mathrm{T}} q_{i}, & & i=0, \ldots, N-1, \\
q_{i} & \geqslant 0, & i & =0, \ldots, N-1 .
\end{array}
$$

In the dual problem, $q_{i}, i=0, \ldots, N-1$, is a multiplier which arises from the primal state-control inequality constraint, equation (17). As an aid to interpretation, we temporarily drop this constraint. In this case, the dual problem may be written:

$$
\operatorname{minimize} \sum_{i=0}^{N-1} c_{i}^{*}\left(y_{i}, v_{i}\right)-a^{\mathrm{T}} z_{-1},
$$

subject to the constraints

$$
\begin{array}{rlrl}
y_{i} & =z_{i-1}-\left(A_{i}^{\mathrm{T}}+1\right) z_{i}, & i=0, \ldots, N-1, \\
y_{N} & =z_{N-1}=A_{N}^{\mathrm{T}} w, & & \\
v_{i} & =-B_{i}^{\mathrm{T}} z_{i}, & i=0, \ldots, N-1 .
\end{array}
$$

Substituting equation (38) into (35) and rearranging equation (36), we have the problem

$$
\operatorname{minimize} \sum_{i=0}^{N-1} \tilde{c}_{i}^{*}\left(y_{i}, z_{i}\right)-a^{T} z_{-1},
$$

subject to the constraints

where

$$
\begin{aligned}
z_{i-1}-z_{i} & =A_{i}^{\mathrm{T}} z_{i}+y_{i}, \quad i=0, \ldots, N-1, \\
y_{N} & =z_{N-1}=A_{N}^{\mathrm{T}} w,
\end{aligned}
$$

$$
\tilde{c}_{i}^{*}\left(y_{i}, z_{i}\right)=c_{i}^{*}\left(y_{i},-B_{i}^{\mathrm{T}} z_{i}\right), \quad i=0, \ldots, N-1 .
$$

In this form, we can readily compare the dual problem with the original unconstrained primal problem given by equations (13), (14), (15) and (16). The term $a^{T} z_{-1}$ captures the original initial condition on the state. From equation (40), we see that $y_{i}$, the dual to the state variable $x_{i}$, acts as a control variable while $z_{i}$ (which is proportional to the dual $v_{i}$, of the control variable $u_{i}$ ) acts as a state 
variable. Further, if the primal problem is a forward time process from initial state $x_{0}$, the dual problem operates in reverse time from the state $z_{N-1}$. Further comment on this interpretation will be given when we make the connection with the Discrete Maximum Principle in the next Section.

We now particularize the objective function in the following two examples.

\section{Example (i). Linear objective}

$$
c_{i}\left(x_{i}, u_{i}\right)=P_{i}^{T} x_{i}+Q_{i}^{T} u_{i},
$$

where $P_{i}$ and $Q_{i}$ are given vectors in $R^{n}$ and $R^{m}$ respectively, for all $i$.

The conjugate transform of $\sum_{i=0}^{N-1} c_{i}\left(x_{i}, u_{i}\right)$ is given by

$$
\begin{aligned}
\sum_{i=0}^{N-1} c_{i}^{*}\left(y_{i}, v_{i}\right) & =\sup _{\substack{\mathbf{X}_{i} \in R^{n} \\
u_{i} \in R^{m}, \forall i}}\left(\sum_{i=0}^{N-1} x_{i}^{\mathrm{T}} y+\sum_{i=0}^{N-1} u_{i}^{\mathrm{T}} v_{i}-P_{i}^{\mathrm{T}} x_{i}-Q_{i}^{\mathrm{T}} u_{i}\right) \\
& =\sup _{x_{i} \in R^{n}, \forall i} \sum_{i=0}^{N-1}\left(y_{i}-P_{i}\right)^{\mathbf{T}} x_{i}+\sup _{u_{i} \in R^{m}, \forall i} \sum_{i=0}^{N-1}\left(v_{i}-Q_{i}\right)^{\mathbf{T}} u_{i} \\
& =0
\end{aligned}
$$

with $y_{i}=P_{i}$ and $v_{i}=Q_{i}, i=0, \ldots, N-1$.

In this case, the dual problem is, from equations (30) to (34),

$$
\text { minimize }-a^{\mathrm{T}} z_{-1}+\sum_{i=0}^{N-1} b_{i}^{\mathrm{T}} q_{i}
$$

subject to the constraints

$$
\begin{aligned}
z_{i-1}-z_{i} & =A_{i}^{\mathrm{T}} z_{i}+P_{i}+E_{i}^{\mathrm{T}} q_{i}, & & i=0, \ldots, N-1, \\
z_{N-1} & =A_{N}^{\mathrm{T}} w, & & \\
Q_{i} & =-B_{i}^{\mathrm{T}} z_{i}-F_{i}^{\mathrm{T}} q_{i}, & & i=0, \ldots, N-1, \\
q_{i} & \geqslant 0, & i & =0, \ldots, N-1 .
\end{aligned}
$$

Example (ii). Quadratic objective

$$
c_{i}\left(x_{i} u_{i}\right)=\frac{1}{2} x_{i}^{\mathrm{T}} P_{i} x_{i}+\frac{1}{2} u_{i}^{\mathrm{T}} Q_{i} u_{i},
$$

where $P_{i}$ and $Q_{i}$ are approximately dimensioned symmetric non-singular matrices.

A routine calculation gives the conjugate transform of $c_{i}\left(x_{i}, u_{i}\right)$ to be

with

$$
\frac{1}{2} y_{i}^{\mathrm{T}} P_{i}^{-1} y_{i}+\frac{1}{2} v_{i}^{\mathrm{T}} Q_{i}^{-1} v_{i}
$$

$$
y_{i}=P_{i} x_{i} \text { and } v_{i}=Q_{i} u_{i}
$$


Hence the dual problem is given by

$$
\text { minimize } \sum_{i=0}^{N-1}\left(\frac{1}{2} y_{i}^{\mathrm{T}} P_{i}^{-1} y_{i}+\frac{1}{2} v_{i}^{\mathrm{T}} Q_{i}^{-1} v_{i}\right)-a^{\mathrm{T}} z_{-1}+\sum_{i=0}^{N-1} b_{i}^{\mathrm{T}} q_{i}
$$

subject to equations (31), (32), (33) and (34).

\section{Relationship with the maximum principle}

A common approach to a discrete time optimal control problem such as the one defined by equations (13), (14), (15) and (16) is via the Maximum Principle [1] which gives necessary conditions for optimality. In the convex case under consideration, these conditions are also sufficient.

THEOREM (the Discrete Maximum Principle). Assume the Kuhn-Tucker constraint qualification is satisfied for equations (14) and (16). Then if $\hat{u}_{0}, \ldots, \hat{u}_{N-1}$ is an optimal control sequence and $\hat{x}_{0}, \ldots, \hat{x}_{N}$ is the corresponding optimal trajectory, there exist costate vectors $\lambda_{0}, \ldots, \lambda_{N} \in R^{n}$ and multiplier vectors $\mu_{0}, \ldots, \mu_{N} \in R^{p}$ such that

$$
\lambda_{i+1}-\lambda_{i}=\nabla_{\hat{x}_{i}} c_{i}\left(\hat{x}_{i}, \hat{u}_{i}\right)-A_{i}^{\mathrm{T}} \lambda_{i+1}-\mu_{i}^{\mathrm{T}} E_{i}, \quad i=0, \ldots, N-1,
$$

which are the costate equations with terminal boundary condition

$$
\begin{aligned}
\lambda_{N} & =A_{N}^{\mathrm{T}} \mu_{N}, \\
-\nabla_{\hat{u}_{i}} c_{i}\left(\hat{x}_{i}, \hat{u}_{i}\right)+B_{i}^{\mathrm{T}} \lambda_{i+1}+\mu_{i}^{\mathrm{T}} F_{i} & =0, \quad i=0, \ldots, N-1,
\end{aligned}
$$

which are the optimality conditions on the Hamiltonian and

$$
\begin{aligned}
\mu_{i} \leqslant 0, & i=0, \ldots, N-1, \\
\mu_{i}^{\mathrm{T}}\left(E_{i} x_{i}+F_{i} u_{i}\right)=0, & i=0, \ldots, N-1 .
\end{aligned}
$$

Moreover, equations (55), (56) and (57) imply the following maximum condition:

$$
-c_{i}\left(\hat{x}_{i}, \hat{u}_{i}\right)+\lambda_{i+1}\left(A_{i} \hat{x}_{i}+B_{i} \hat{u}_{i}\right) \geqslant-c_{i}\left(\hat{x}_{i}, u_{i}\right)+\lambda_{i+1}\left(A_{i} \hat{x}_{i}+B_{i} u_{i}\right)
$$

for all $u_{i}$, satisfying equation (14). Hence the term Maximum Principle.

Proof. See for example, [1].

We note that the Maximum Principle approach requires the simultaneous solution of the state equations (14), the costate equations (53) and the optimality conditions (55). Further, we note that the boundary conditions, equations (15) and (54) are split between times 0 and $N$. Hence, in general, one must solve a two-point boundary value problem: a problem of not inconsiderable difficulty. 
We now make the connection between this approach and the dual problem as outlined in Section 3. From equation (12), we may relate the primal variables $u_{i}$ and $x_{i}$ with their corresponding dual variables $v_{i}$ and $y_{i}$, for all $i$. This gives us that

and

$$
v_{i}=\nabla_{u_{i}} c_{i}\left(x_{i}, u_{i}\right), \quad i=0, \ldots, N-1 \text {, }
$$

$$
y_{i}=\nabla_{x_{i}} c_{i}\left(x_{i}, u_{i}\right), \quad i=0, \ldots, N-1 \text {, }
$$

at optimaiity. Substituting resuits (59) and (60) into the dual constraints, equations (31) and (33), we obtain

and

$$
\nabla_{u_{i}} c_{i}\left(x_{i}, u_{i}\right)=-B_{i}^{\mathrm{T}} z_{i}-F_{i}^{\mathrm{T}} q_{i}, \quad i=0, \ldots, N-1,
$$

$$
\nabla_{x_{i}} c_{i}\left(x_{i}, u_{i}\right)=z_{i-1}-\left(A_{i}^{\mathrm{T}}+I\right) z_{i}-E_{i}^{\mathrm{T}} q_{i}, \quad i=0, \ldots, N-1 .
$$

If we set $z_{i-1}=-\lambda_{i}$ and $q_{i}=-\mu_{i}$, for any $i$, in equations (44) and (45), we obtain costate equations (53) and the optimality conditions, equations (54), of the Maximum Principle approach. Hence we identify the dual variables $z_{i}$, $i=0, \ldots, N-1$, with the costate variables and the other dual variables $q_{i}$, $i=0, \ldots, N-1$, with the constraint multipliers. The dual problem is then a minimization over constraints which represent the costate equations and the optimality conditions on the Hamiltonian of the Maximum Principle approach.

\section{References}

[1] M. Canon, D. Cullum and E. Polak, Theory of optimal control and mathematical programming (McGraw-Hill, New York, 1970).

[2] P. Kleindorfer, C. Kriebels, G. Thompson and G. Kleindorfer, "Discrete optimal control of production plans", Management Science 22 (1975), 261-273.

[3] M. Kohn and Y. Plessner, "An applicable model of optimal marketing policy", Operations Research 21 (1973), 401-412.

[4] E. L. Peterson, “Geometric programming”, SIAM Review 18 (1976), 1-52.

School of Mechanical and Industrial Engineering

University of New South Wales

Kensington, N.S.W. 2033 